\title{
BMJ Global Health Learning from LMICs: best practices for leveraging sentinel surveillance systems to track the US COVID-19 pandemic
}

\author{
Heather Root (D , ${ }^{1}$ Ross Boyce, ${ }^{1}$ Whitney R Robinson ${ }^{2,3}$
}

To cite: Root $\mathrm{H}$, Boyce R, Robinson WR. Learning from LMICs: best practices for leveraging sentinel surveillance systems to track the US COVID-19 pandemic. BMJ Global Health 2020;5:e004685. doi:10.1136/ bmjgh-2020-004685

Received 7 December 2020 Revised 11 December 2020 Accepted 15 December 2020

Check for updates

(C) Author(s) (or their employer(s)) 2020. Re-use permitted under CC BY-NC. No commercial re-use. See rights and permissions. Published by BMJ.

${ }^{1}$ Department of Medicine, Division of Infectious Diseases, University of North Carolina at Chapel Hill School of Medicine, Chapel Hill, North Carolina, USA ${ }^{2}$ Department of Epidemiology, University of North Carolina at Chapel Hill Gillings School of Global Public Health, Chapel Hill, North Carolina, USA

${ }^{3}$ University of North Carolina at Chapel Hill Carolina Population Center, Chapel Hill, North Carolina, USA

Correspondence to Dr Whitney R Robinson; whitney_robinson@unc.edu

\section{FRAMING}

How is the USA like a low-income and middle-income countries (LMICs)? With limited treatment options, no vaccine and a chronically underfunded public health infrastructure, the USA is confronting the COVID-19 pandemic hampered by adversities typically faced by LMICs. In particular, obtaining reliable and timely surveillance data to inform public health policy has been impeded by persistent shortages of testing supplies and inequitable access to testing. SARS-CoV-2 testing in March and April 2020 likely identified only $2 \%-17 \%$ of US infections. ${ }^{1}$

These deficiencies in testing mean that the USA lacks accurate surveillance data for tracking SARS-CoV-2 outbreaks in its communities. LMICs long ago confronted and adapted to similar difficulties by pioneering 'sentinel surveillance' systems. Sentinel surveillance is the study of disease rates in a specific cohort to estimate trends in a larger population. When properly implemented, sentinel surveillance systems offer an effective method for leveraging limited resources to monitor disease spread. In this commentary, we argue that rigorously designed and thoughtfully implemented sentinel surveillance systems are a cost-effective and logistically viable strategy to monitor local SARS-CoV-2 transmission in the contemporary USA. We use the example of HIV sentinel surveillance in sub-Saharan Africa during the early decades of the AIDS epidemic as an exemplar.

\section{OVERVIEW}

Sentinel surveillance systems serve multiple functions. The method enables prompt and flexible monitoring and investigation of suspected public health problems and the ability to target public health interventions
Summary box

With limited treatment options, no vaccine and a chronically underfunded public health infrastructure, the USA is confronting the COVID-19 pandemic hampered by adversities typically faced by low-income and middle-income countries (LMICs).

- LMICs long ago confronted and adapted to similar difficulties by pioneering 'sentinel surveillance' systems.

- Sentinel surveillance is the study of disease rates in a specific cohort to estimate trends in a larger population.

- When properly implemented, sentinel surveillance systems offer an effective method for leveraging limited resources to monitor disease spread.

- In this viewpoint, we describe five key features that characterise the most successful sentinel surveillance programmes and argue that rigorously designed and thoughtfully implemented sentinel surveillance systems are a cost-effective and logistically viable strategy to monitor local SARS-CoV-2 transmission in the contemporary USA

- We use the example of HIV sentinel surveillance in sub-Saharan Africa during the early decades of the AIDS epidemic as an exemplar.

more effectively. ${ }^{2}$ Benefits include early warnings of growing outbreaks, quantification of the size and scope of an epidemic, short-term or long-term evaluation of control efforts and stimulating political and social action. Sentinel surveillance can identify behavioural risk factors associated with transmission as well as identify core transmitters (reservoirs); estimate prevalence and incidence trends in specific demographic risk groups and geographic areas; and help anticipate future resources needed to cope with an epidemic. However, some sentinel surveillance systems are more accurate and functional than others. Below we describe five key features that characterise the most successful sentinel surveillance programmes. 


\section{FIVE CONSIDERATIONS FOR A SUCCESSFUL SENTINEL SURVEILLANCE PROGRAMME}

First, the sentinel population should be representative of some high-risk target population or generate prevalence or incidence rates that correlate with rates of key, defined target populations. For instance, during the HIV epidemic of the late 1980s, public health officials working in LMICs popularised the strategy of testing leftover, or 'remnant', blood from routine syphilis testing among pregnant women attending antenatal clinics (ANCs). In the sub-Saharan Africa of the 1980s and 1990s, heterosexual vaginal intercourse was a dominant mode of HIV transmission. ${ }^{3}$ Thus, many countries chose patients with sexually transmitted infection (STI) and ANC attendees as their sentinel groups to monitor HIV seroprevalence. ${ }^{4}$ A convenience sample of low-risk people, for example, elderly people shopping at the market, would have failed to reflect HIV prevalence meaningfully. An added benefit of ANC serosurveillance is the potential identification of HIV-positive pregnant people, a clinically relevant population eligible for intervention to prevent mother-to-child HIV transmission.

Second, an effective sentinel surveillance system should engage an easily accessible population and be economical. During the late 1980s, WHO's Global Program on AIDS and affiliated research institutions developed HIV sentinel surveillance in large part because of the high costs of other potentially suitable methods such as population-based survey studies. Instead, as described above, researchers analysed remnant blood samples from ANC attendees: antenatal clinics were easy to locate, represented the sentinel population well and were accessible to researchers. Furthermore, many developing countries adopted a strategy known as unlinked anonymous testing (UAT) ${ }^{5}$ Patients with ANC were neither consented nor told the results of their HIV tests. UAT dramatically simplified the logistics of and reduced the price of ANC sentinel serosurveillance. Additionally, because of stigma around HIV testing in that era, UAT likely reduced biases that would have been present with voluntary testing. However, UAT also raises ethical concerns, as discussed later.

Third, a sentinel surveillance system should be able to test predetermined population groups in a regular, consistent and sustainable way. ${ }^{4}$ In monitoring HIV infection, when countries were obtaining information from ANC and STI clinics, the sentinel groups, sites and study procedures became established and regular. ${ }^{4}$ Consistent and predictable data generation created an infrastructure that allowed results to be used rapidly and confidently in HIV-related public health decision making.

A fourth characteristic of an effective sentinel surveillance system is the extent to which it signals changes in transmission dynamics. For example, if HIV transmission in sub-Saharan Africa began to be dominated by blood transfusions or intravenous drug use, then ANC serosurveillance would decrease in its utility. Relatedly, well-defined populations with unique risk profiles may reflect part of transmission dynamics while missing other changes in transmission. Therefore, triangulating among multiple sentinel groups or continuously evaluating the performance of current sentinel groups will enhance surveillance effectiveness.

Finally, one must consider the ethics of sentinel surveillance, especially systems that do not report individualised, or 'named', test results back to those tested. For instance, UAT-based ANC HIV serosurveillance was constantly questioned on ethical grounds. Some viewed this strategy as inconsistent with the core bioethical principle of respect for persons. Not consenting patients withdraws the autonomy of a pregnant woman to make informed choices about participating in surveillance-related testing. ${ }^{3}$ It is important to note that the ethical balance of UAT approaches will change as treatment and transmission prevention options evolve. UAT becomes more ethically untenable once results of tests have actionable prevention or treatment implications. As well-tolerated treatment options for HIV became more available globally, UAT policy changed as a result.

\section{SENTINEL SURVEILLANCE AND COVID-19}

Recently published studies analyse seroprevalence of SARS-CoV-2 using UAT approaches. All these studies meet two criteria described above. First, they are accessible, timely and economical. Second, because seroprevalence is of limited clinical utility for most people and named testing is largely available in the USA when clinically indicated, we believe UAT serosurveillance is ethically justified. However, some recent studies fall short on the three other criteria described: inadequately justified sentinel groups; relying on cross-sectional data rather than consistent, sustainable sampling over time; and not evaluating how well results reflect actual prevalence or transmission dynamics. ${ }^{16}$ Flannery $e t a l^{7}$ improve on other studies by selecting a well-defined sentinel group: hospitalised delivering babies. While Stadlbauer et at argues that pregnant people may under-represent prevalence, it is just as likely that parturient patients are at average or high risk because reproductive-aged women have many social contacts, are disproportionately likely to be 'essential workers' in caregiving fields and socialise with other young people who may unwittingly transmit the virus because they are asymptomatic or have mild symptoms. The utility of different sentinel populations in representing SARS-CoV-2 prevalence and transmission is an unsettled question that demands empirical research.

\section{CONCLUSION}

LMICs have long contended with infectious diseases threats and limited treatment and prevention resources. Communities in the USA now find themselves in a situation where they can learn from the ingenuity and successes of LMICs. Monitoring local SARS-CoV-2 transmission through sentinel surveillance will allow public officials to relax and tighten public health restrictions 
in a targeted manner that may minimise public fatigue and hardship while also minimising transmission. Even as COVID-19 vaccines are rolled out throughout the USA, ${ }^{89}$ sentinel surveillance can also help monitor the effectiveness of vaccination programmes in the sentinel population. Thoughtfully designed sentinel surveillance systems are a cost-effective part of a larger strategy to mitigate the spread of the COVID-19 pandemic in resource-limited settings.

Acknowledgements We would like to thank Allison Aiello and other collaborators on the North Carolina Network Surveillance Studies.

Contributors All three authors contributed substantially to the conception and design of the work, to drafting the work, to revising the work critically for important intellectual content and to final approval of the version published. All three authors are in agreement to be accountable for all aspects of the work in ensuring that questions related to the accuracy and integrity of any part of the work are appropriately investigated and resolved.

Funding WRR receives support from Gillings Innovations Labs, Gillings School of Global Public Health, University of North Carolina at Chapel Hill. RB receives support from the National Institutes of Health (K23Al141764) and the North Carolina Department of Health and Human Services through the North Carolina Partnership for Excellence in Applied Epidemiology programme.

Competing interests None declared.

Patient consent for publication Not required.

Provenance and peer review Not commissioned; internally peer reviewed.

Data availability statement There are no data in this work.

Open access This is an open access article distributed in accordance with the Creative Commons Attribution Non Commercial (CC BY-NC 4.0) license, which permits others to distribute, remix, adapt, build upon this work noncommercially, and license their derivative works on different terms, provided the original work is properly cited, appropriate credit is given, any changes made indicated, and the use is non-commercial. See: http://creativecommons.org/ licenses/by-nc/4.0/.

ORCID iD

Heather Root http://orcid.org/0000-0003-2293-0033

\section{REFERENCES}

1 Havers FP, Reed C, Lim TW. Seroprevalence of antibodies to SARSCoV-2 in six sites in the United States. medRxiv 2020

2 Nsubuga P, White ME, Thacker SB, World Bank. Public Health Surveillance: A Tool for Targeting and Monitoring Interventions. In: Jamison DT, Breman JG, Measham AR, eds. Disease control priorities in developing countries. 2nd ed, 2006. http://www.ncbi.nlm.nih.gov/ books/NBK11770/

3 Dee J, Garcia Calleja JM, Marsh K, et al. Hiv surveillance among pregnant women attending antenatal clinics: evolution and current direction. JMIR Public Health Surveill 2017;3:e85.

4 Slutkin G, Chin J, Tarantola D. Sentinel surveillance for HIV infection: a method to monitor HIV infection trends in population groups. World Health Organization, 1988. http://apps.who.int/iris/bitstream/10665/ 61728/1/WHO GPA DIR 88.8.pdf

5 GTZ. HIV/AIDS surveillance in developing countries, 1999. Available: https://www.who.int/hiv/strategic/surveillance/en/GTZ surveillance. pdf?ua=1 [Accessed 25 May 2020].

6 Stadlbauer D, Tan J, Jiang K. Seroconversion of a city: longitudinal monitoring of SARS-CoV-2 seroprevalence in New York City. medRxiv 2020.

7 Flannery DD, Gouma S, Dhudasia MB. SARS-CoV-2 seroprevalence among parturient women. medRxiv 2020. [Epub ahead of print: $10 \mathrm{Jul}$ 2020]. doi:10.1101/2020.07.08.20149179

8 Pfizer. Pfizer and BioNTech to submit emergency use Authorization Request today to the U.S. FDA for COVID-19 vaccine, 2020. Available: https://www.pfizer.com/news/press-release/press-releasedetail/pfizer-and-biontech-submit-emergency-use-authorization

9 Moderna, Inc. Moderna Announces primary efficacy analysis in phase 3 COVE study for its COVID-19 vaccine candidate and filing today with U.S. FDA for emergency use Authorization, 2020. Available: https://investors.modernatx.com/news-releases/news-release-details/ moderna-announces-primary-efficacy-analysis-phase-3-cove-study/ 